\title{
Some results on optimal control of stochastic systems with state chance constraints
}

\author{
Zahia Bouabbache $^{1}$, Eric Busvelle ${ }^{2}$ and Mohamed Aidene ${ }^{1}$
}

\begin{abstract}
We consider a continuous-time control problem with random initial condition and chance constraints. We solve this problem by discretizations and we prove that the discretetime problem is convex and can be solved by the method of the logarithmic barrier function. Then we prove that when the discretization step goes to zero, the cost of the solutions of the discrete-time problems converge to the optimal cost of the continuous-time problem.
\end{abstract}

\section{INTRODUCTION}

In this paper, we consider a feedforward optimal control problem with stochastic initial state and stochastic constraints (called in many papers "chance constraints", see [1] for an excellent review or [2] for some results related to our works).

Chance constraints in optimal control problems are a powerful formalism to deal with realistic constraints. In many cases, constraints are inherently stochastic constraints, see [3]-[5]. Moreover, chance constraints are less binding than deterministic constraints and may encourage more smooth control (see our example for instance).

In order to solve numerically this problem, we present a finite-dimensional stochastic problem which is closely related to the continuous time version. This problem belongs to a class of stochastic optimal control problem which is more general than the continuous-time problem but that can be solved easily with a standard method (Logarithmic Barrier Function Method - SUMT, see [6], [7]), adapted to a convex optimization problem. Therefore, we will prove that our problem is convex. One of the main originality of this work comes from the fact that we consider a degenerate normal distribution, which is not absolutely continuous w.r.t. Lebesgue measure. We show that despite this, our problem remains convex and we describe a numerical method to solve this problem.

Our main objective in this work is to deal with continuoustime optimization problem. Thererfore, in the second part of this paper, we present the continuous-time version of our optimization problem and we show that the solution can be approximated by solving the discrete-time problem as explained in the first part.

Let us remark that our optimal control problems are fixed final time problems. This is a suitable approach when one want to consider finite-horizon stochastic model predictive control problems ( [3]-[5], [8]).

In the last part, we present a short example to illustrate the method.

\footnotetext{
${ }^{1}$ L2CSP, Université Mouloud Mammeri de Tizi Ouzou, Algérie. Corresponding author: bouabbache_zahiadyahoo.fr

${ }^{2}$ Aix Marseille Univ., Université de Toulon, CNRS, ENSAM, LSIS, Marseille, France
}

\section{DISCRETE-TIME PROBLEM}

\section{A. Statement of the problem}

We consider the following linear discrete-time optimal control problem:

$$
\left\{\begin{array}{l}
J(u)=E\left[\frac{1}{2} x(N)^{\prime} C x(N)+c x(N)\right] \longrightarrow \min _{u} \\
x(n+1)=A(n) x(n)+B(n) u(n)+D(n) \boldsymbol{W}(n) \\
x(0)=\boldsymbol{X} \\
\mathbb{P}(\{\forall n=1, \ldots, N ; H(n) x(n) \leq g(n)\}) \geq \alpha \\
u^{-} \leq u(n) \leq u^{+}
\end{array}\right.
$$

for a given integer $N, n=0$ to $N$, where matrices have the following dimensions : $A \in \mathbb{R}^{n_{x} \times n_{x}}, B \in \mathbb{R}^{n_{x} \times n_{u}}$, $D \in \mathbb{R}^{n_{x} \times n_{x}}, C \in \mathbb{R}^{n_{x} \times n_{x}}, c \in \mathbb{R}^{1 \times n_{x}}, H \in \mathbb{R}^{n_{c}(n) \times n_{x}}$ of rank $n_{c}(n) \leq n, g \in \mathbb{R}^{n_{c}(n)}, J(u)$ is the objective function. The number of constraints $n_{c}$ may depend of $n$ (as in Section V).

$x(n) \in \mathbb{R}^{n_{x}}$ in eq. (1b) is the state of a controlled system and $u(n) \in \mathbb{R}^{n_{u}}$ is the control. The control function $u(n)$ is assumed to be a measurable and bounded function with lower bound $u^{-}$and upper bound $u^{+}$. In this paper, vector inequalities have the usual interpretation, hence eq. (1e) means that $u_{j}^{-} \leq u_{j}(n) \leq u_{j}^{+}$for $j=1, \ldots, n_{u}$. The vector inequality $H(n) x(n) \leq g(n)$ is also defined component by component in $\mathbb{R}^{n_{c}(n)}$.

$\boldsymbol{X}$ is a random variable and it is the only stochastic parameter in this problem. $\boldsymbol{X}$ is supposed to be normally distributed $\boldsymbol{X} \sim \mathcal{N}(\mu, \Sigma)$ and this gaussian distribution may be degenerated ( $\Sigma$ is not supposed definite positive), see Section II-B. $\boldsymbol{W}$ is a white noise process, independent from $\boldsymbol{X}(\boldsymbol{W}(n) \sim \mathcal{N}(0, I)$ and $\boldsymbol{W}(j)$ is independent from $\boldsymbol{W}(k)$ if $j \neq k)$.

This problem is an optimal linear control problem in discrete time with chance constraints. In the following, we suppose that the set of solutions is not empty.

\section{B. Degenerated Gaussian law}

Matrices $\Sigma, D(n)$ and $H(n)$ are not supposed to be invertible. For instance, the deterministic case is a particular case of our settings. If $\Sigma$ is a singular matrix, the corresponding problem is a stochastic problem with initial condition belonging to a submanifold of $\mathbb{R}^{n_{x}}$. It may also happen that $D(n)=0$ (no state noise) or $H(n)=0$ except for $n=N$ which correspond to a final chance constraint. Anyway, even if these matrices are invertible, our method to solve the problem will consist in some linear change 
of variables which is not necessarily invertible. Therefore, in order to prove some convexity results concerning our problem, it is necessary to clearly define degenerate Gaussian laws.

We use the extension of Gaussian density function to the degenerate case as explained in [9]. Briefly, let $\boldsymbol{Y}$ be a degenerate Gaussian random variable with mean $m$ and covariance matrix $Q$.

Let us write $Q=\sum_{i=1}^{n} \mu_{i} U_{i} U_{i}^{T}$ the spectral expansion of $Q$ where $\mu_{i}$ and $U_{i}$ are respectively the eigenvalues and the eigenvectors of $Q$ ( [10]). Let us denote $Q^{-}=\sum_{i=1}^{r} \mu_{i}^{-1} U_{i} U_{i}^{T}$ its Moore-Penrose inverse where $\mu_{r+1}, \ldots, \mu_{n}$ are the eigenvalues equals to zero. Then the distribution of $\boldsymbol{Y}$ may be written:

$$
\begin{aligned}
f(\boldsymbol{Y})= & (2 \pi)^{-\frac{r}{2}}\left(\prod_{i=1}^{r} \mu_{i}\right)^{-\frac{1}{2}} \\
& \exp \left(-\frac{1}{2}(\boldsymbol{Y}-m)^{T} Q^{-}(\boldsymbol{Y}-m)\right) \\
& \prod_{i=r+1}^{n} \delta\left((\boldsymbol{Y}-m)^{T} U_{i}\right)
\end{aligned}
$$

Therefore, this distribution is supported by the set $E=$ $\bigcap_{i=r+1}^{n}\left\{\boldsymbol{Y} \in \mathbb{R}^{n} ;(\boldsymbol{Y}-m)^{T} U_{i}=0\right\}$ and the probability of a subset $A \subset \mathbb{R}^{n}$ if equal to

$$
\begin{array}{r}
\mathbb{P}_{\boldsymbol{Y}}(A) \\
=\frac{1}{D} \int_{A \cap E} \exp \left(-\frac{1}{2}(\boldsymbol{Y}-m)^{T} Q^{-}(\boldsymbol{Y}-m)\right) d \lambda_{/ E} \boldsymbol{Y} \\
\stackrel{\text { def. }}{=} \mathbb{P}_{\boldsymbol{Y} / E}(A \cap E)
\end{array}
$$

where $\lambda_{/ E}$ is the Lebesgue measure restricted to $E$. $D$ is the normalization factor explicitly written in (2), and $f_{E}(\boldsymbol{Y})=$ $\frac{1}{D} \exp \left(-\frac{1}{2}(\boldsymbol{Y}-m)^{T} Q^{-}(\boldsymbol{Y}-m)\right)$ is the Radon-Nikodym derivative of $\mathbb{P}_{\boldsymbol{Y} / E}$ w.r.t. $\lambda_{/ E}$ which exists since $P_{\boldsymbol{Y} / E} \ll$ $\lambda_{/ E} \cdot \mathbb{P}_{\boldsymbol{Y} / E}$ is a non degenerate Gaussian distribution and $f_{E}$ its density function.

Let us remark that $E$ is an affine subspace of $\mathbb{R}^{n}$.

\section{RESOLUTION}

\section{A. Problem transformation}

As usual for linear time-dependent system, eq. (1b) can be written for $0 \leq n \leq N$ :

$$
\begin{aligned}
x(n)=\phi(n, 0) \boldsymbol{X} & +\sum_{k=0}^{n-1} \phi(n, k+1) B(k) u(k) \\
& +\sum_{k=0}^{n-1} \phi(n, k+1) D(k) \boldsymbol{W}(k)
\end{aligned}
$$

where $\phi(n, k)=A(n-1) A(n-2) \cdots A(k)$ or by recurrence

$$
\left\{\begin{aligned}
\phi(n+1, k) & =A(n) \phi(n, k) \\
\phi(k, k) & =I d
\end{aligned}\right.
$$

Using eq. (4) for $n=N$, thanks to the fact that $\boldsymbol{W}$ is a white noise independent from $\boldsymbol{X}$, eq. (1a) can be rewritten as a quadratic expression in $u$ :

$$
J(u)=\frac{1}{2} \bar{u}^{\prime} \bar{C} \bar{u}+\bar{c} \bar{u}+\text { constant }
$$

where $^{1}$

- $\bar{u}=(u(0) ; \cdots ; u(N-1))$

- $\bar{c}=c+b$

- $\bar{C}=b^{\prime} C b$

and $b=(\phi(N, 1) B(0) ; \cdots ; \phi(N, N) B(N-1))$.

Using eq. (4), one can rewrite eq. (1d) explicitly:

$$
\mathbb{P}(\{\bar{B} \bar{u} \leq \bar{g}-\bar{A} \boldsymbol{X}-\bar{D} \overline{\boldsymbol{W}}\}) \geq \alpha
$$

where

- $\bar{W}=(\boldsymbol{W}(0), \ldots, \boldsymbol{W}(N-1))^{\prime}$

- $\bar{g}=(g(1) ; \ldots ; g(N))$

- $\bar{A}=(H(1) \phi(1,0) ; \ldots ; H(N) \phi(N, 0))^{\prime}$

- $\bar{B}$ (respectively $\bar{D}$ ) is a block lower triangular matrix where each block is defined as $\bar{B}_{n, k}=$ $H(n) \phi(n, k) B(k-1)$ for $n, k=1, \cdots, N$ (resp. $\bar{D}_{n, k}=H(n) \phi(n, k) D(k-1)$

Then, using eq. (6) and eq. (7), the problem eq. (1a) to eq. (1e) becomes

$$
\left\{\begin{array}{l}
J(\bar{u})=\frac{1}{2} \bar{u}^{\prime} \bar{C} \bar{u}+\bar{c} \bar{u} \longrightarrow \min _{u} \\
\mathbb{P}(\{\bar{B} \bar{u} \leq \boldsymbol{Y}\}) \geq \alpha \\
\bar{u}^{-} \leq \bar{u} \leq \bar{u}^{+}
\end{array}\right.
$$

where $\bar{u}^{-}=\left(u^{-} ; \cdots ; u^{-}\right)$and same for $\bar{u}^{+}$, and where $\boldsymbol{Y}=$ $\bar{g}-\bar{A} \boldsymbol{X}-\bar{D} \overline{\boldsymbol{W}}$ is a possibly degenerate Gaussian random variable (indeed, even if $\boldsymbol{X}$ is a non-degenerate Gaussian random variable, it may not be the case for $\boldsymbol{Y}$ ).

Let $\mathbb{P}_{\boldsymbol{Y}}$ denote the probability of $\boldsymbol{Y}$, which is defined as in Section II-B.

\section{B. Convexity}

We claim that our optimization problem is a convex optimization problem.

First of all, the function to minimize is the sum of a convex quadratic function and a linear function and therefore is convex. In the following, we will denote this function $J(\bar{u})$ and the method can be applied to any convex function $J(\bar{u})$.

We want to prove that the set of admissible controls (satisfying our constraints) is a convex subset of $\mathbb{R}^{N}$.

Lemma 1: The probability measure $\mathbb{P}_{\boldsymbol{Y}}$ is log-concave.

Proof: We want to show that $\mathbb{P}_{\boldsymbol{Y}}(\alpha A+(1-\alpha) B) \geq$ $\mathbb{P}_{\boldsymbol{Y}}(A)^{\alpha} \mathbb{P}_{\boldsymbol{Y}}(B)^{1-\alpha}$ for any convex subsets $A, B \subset \mathbb{R}^{n}$ and any $0 \leq \alpha \leq 1$. But $\mathbb{P}_{\boldsymbol{Y}}(\alpha A+(1-\alpha) B)=\mathbb{P}_{\boldsymbol{Y} / E}(\alpha A \cap$ $E+(1-\alpha) B \cap E)$. Moreover $\mathbb{P}_{\boldsymbol{Y} / E}$ if a non-degenerate Gaussian distribution and its density function is log-concave and therefore $\mathbb{P}_{\boldsymbol{Y} / E}$ is log-concave (see for instance [6]

\footnotetext{
${ }^{1}(M(0) ; \cdots ; M(N))$ where $M(n)$ are matrices (or vectors), each of them having the same number of columns, is the block-matrix (or vector) built by stacking each matrix in a single column (as in Matlab notation)
} 
Theorem 2.1). Since $A \cap E$ and $B \cap E$ are convex as the intersection of convex subsets of $\mathbb{R}^{n}, \mathbb{P}_{\boldsymbol{Y} / E}(\alpha A \cap E+(1-$ $\alpha) B \cap E) \geq \mathbb{P}_{\boldsymbol{Y} / E}(A \cap E)^{\alpha} \mathbb{P}_{\boldsymbol{Y} / E}(B \cap E)^{1-\alpha}$ and this concludes the proof since $\mathbb{P}_{\boldsymbol{Y} / E}(A \cap E)=\mathbb{P}_{\boldsymbol{Y}}(A)$ and $\mathbb{P}_{\boldsymbol{Y} / E}(B \cap E)=\mathbb{P}_{\boldsymbol{Y}}(B)$.

Remark 1: Our Lemma 1 is a generalization of Theorem 2.1 in [6] to a probability which is not absolutely continuous w.r.t. Lebesgue measure.

Theorem 1: The minimization problem described by eq. (8a) to eq. (8c) is convex.

Proof: $J$ being convex, the set $u^{-} \leq \bar{u} \leq u^{+}$being a convex subset of $\mathbb{R}^{N}$, it suffices to prove that the constraint $\{G(\bar{u}) \geq \alpha\}$, where $G(\bar{u})=\mathbb{P}(\{\bar{B} \bar{u} \leq \boldsymbol{Y}\})$, induces a convex constraint on $u$.

In [6], it is shown (Theorem 2.1) that if $\boldsymbol{Y}$ is a random variable that has logconcave probability distribution and $\mathrm{g}(\mathrm{u}, \mathrm{y})$ is a concave (or quasi-concave) function of the variables $u \in \mathbb{R}^{n_{u}}$ and $y \in \mathbb{R}^{n_{y}}$ then the function $G(u)=$ $\mathbb{P}(\{g(u, \boldsymbol{Y}) \geq 0\}), u \in \mathbb{R}^{n_{x}}$ is logconcave. Thanks to this theorem and lemma $1, G(\bar{u})$ is logconcave and so it is also quasi-concave. Therefore, $\{G(\bar{u}) \geq \alpha\}=G^{-1}([\alpha,+\infty[)$ is convex. This proves that our optimization problem is convex.

\section{NUMERICAL RESOLUTION}

Our optimization problem is convex but $G(\bar{u})=\mathbb{P}(\{\bar{B} \bar{u} \leq$ $\boldsymbol{Y}\}$ ) have no continuous gradients (and may be not continuous). However, since $G(\bar{u})$ is a logconcave function, a suitable method in this case is to use a sequential unconstrained minimization technique (SUMT) using a logarithmic barrier function, as described in [6] and [7].

Let us describe briefly the logarithmic barrier function method. First of all, the problem is converted to the following problem:

$$
\begin{array}{r}
\min _{\bar{u}} J(\bar{u})-\theta \log (G(\bar{u})-\alpha) \\
\text { subject to } u^{-} \leq \bar{u} \leq u^{+}
\end{array}
$$

Since $J$ is convex, $G$ is logconcave, and the domain is a bounded set, the objective function 9 is convex for any $\theta$. Moreover, the solution $\bar{u}_{\theta}$ of this problem is such that

$$
\lim _{\theta \rightarrow 0} \bar{u}_{\theta}=\bar{u}^{*}
$$

where $\bar{u}^{*}$ is the solution of our problem ( [7]).

The main difficulty in the resolution of this problem is the estimation of the probability $\mathbb{P}(\{\bar{B} \bar{u} \leq \boldsymbol{Y}\})$ for a degenerated normal distribution. We used a Monte-Carlo method to estimate this integral with Sobol pseudo-random numbers over $[0,1]^{r}, r$ being the dimension of the linear subspace $E=\operatorname{supp}(\mathbb{P})$. We used Box-Muller method to simulate the Gaussian law and we estimate $\mathbb{P}(\{\bar{B} \bar{u} \leq \boldsymbol{Y}\})$ by the frequency of pseudo-random realizations satisfying $\bar{B} \bar{u} \leq \boldsymbol{Y}$.

\section{CONTINUOUS-TIME PROBLEM}

\section{A. Statement of the problem}

We consider the following linear fixed-final-time optimal control problem on the interval $\left[t_{0}, T\right]$

$$
\left\{\begin{array}{l}
J(u)=E[c x(T)] \longrightarrow \min _{u} \\
\dot{x}(t)=A x(t)+B u(t) \\
x\left(t_{0}\right)=\boldsymbol{X} \\
\mathbb{P}(\{H x(T) \leq g\}) \geq \alpha \\
u^{-} \leq u(t) \leq u^{+}
\end{array}\right.
$$

where matrices have the same dimensions as in eqs. (1a) to $(1 \mathrm{e})$. Besides the fact that this problem is in continuous time, the main differences between this optimization problem and its discrete-time counterpart are:

- The cost to minimize in eq. (10a) is linear,

- There is no more state noise, the only random variable is $\boldsymbol{X} \sim \mathcal{N}(\mu, \Sigma)$ where $\Sigma$ is supposed to be definite positive,

- There is no more chance constraints along the trajectory, the chance constraint concerns only the state at fixed final time $T$.

We didn't try to generalize the continuous-time problem to a broad class of problems since our objective is to compare this approach to a new approach which is currently in preparation and which concerns a method devoted to this particular case of continuous-time problems.

Let us simplify the problem eqs. (10a) to (10e). First of all, one can write

$$
x(T)=\mathrm{e}^{A T} \boldsymbol{X}+\int_{0}^{T} \mathrm{e}^{A(T-s)} B u(s) d s
$$

so that eq. (10a) and eq. (10d) may be rewritten

$$
J(u)=c \mathrm{e}^{A T} \mu+c F(u)
$$

and

$$
\mathbb{P}\left(H F(u) \leq g-H \mathrm{e}^{A T} \boldsymbol{X}\right) \geq \alpha
$$

respectively, where $F(u)$ is the linear functional

$$
F(u)=\int_{0}^{T} \mathrm{e}^{A(T-s)} B u(s) d s
$$

Let $\Omega(u)=\left\{F(u) ; u \in L^{\infty}\left(\left[u^{-}, u^{+}\right]\right)\right\}$. The set $\Omega(u) \subset$ $\mathbb{R}^{n_{x}}$ is the accessibility set starting from $x\left(t_{0}\right)=0$ and driven by eq. (10b).

The problem described by eqs. (10a) to (10e) is clearly equivalent to the two following subproblems:

1) minimize $c \xi$ over $\xi \in \Omega(u)$ and under the constraint $\mathbb{P}\left(H \xi \leq g-H \mathrm{e}^{A T} \boldsymbol{X}\right) \geq \alpha$

2) and then find $u$ such that $F(u)=\xi$.

Let $\xi^{*}$ denotes a solution to the first problem and $J^{*}=$ $c \mathrm{e}^{A T} \mu+c \xi^{*}$ the corresponding optimal cost. Therefore, the second problem is just a control problem (find $u$ to send $x(t)$ from $x\left(t_{0}\right)=0$ to $\left.x(T)=\xi^{*}\right)$. So $J\left(u^{*}\right)=J^{*}$ and one can assume $u$ piecewise continuous. 


\section{B. Link to the discrete-time problem}

In the following, we denote $\Phi(u)$ the nonlinear function $\Phi(u): u \mapsto \mathbb{P}\left(H F(u) \leq g-H \mathrm{e}^{A T} \boldsymbol{X}\right)$.

We consider a collection of discrete-time problems corresponding to discretizations of eqs. (10a) to (10e). Indeed, let us choose a discretization step $T_{k}=\frac{T}{N_{k}}$ for $k=1,2, \ldots$, $N_{k}$ being a growing unbounded sequence of integers. Let us denotes $A_{k}$ and $B_{k}$ the two matrices corresponding to the discretization of eq. (10b), that is

$$
\begin{aligned}
& A_{k}=\mathrm{e}^{A T_{k}} \\
& B_{k}=\int_{0}^{T_{k}} \mathrm{e}^{A\left(T_{k}-s\right)} B d s
\end{aligned}
$$

and set $A(n)=A_{k}, B(n)=B_{k}$ and $D(n)=0$ in the discrete-time dynamic system eq. (1b). We set $C=0$ in eq. (1a) and $H(n)=0, g(n)=0$ in eq. (1d) except $H\left(N_{k}\right)$ and $g\left(N_{k}\right)$ which are equals to $H$ and $g$ respectively $\left(N_{k}\right.$ is the sample corresponding to time $N_{k} T_{k}=T$ ).

Theorem 2: For any $\varepsilon>0$ and $\eta>0$, let us denotes $u_{k}^{*}$ an optimal solution of the discrete-time problem:

$$
\left\{\begin{array}{l}
J(u)=E\left[c x\left(N_{k}\right)\right] \longrightarrow \min _{u} \\
x(n+1)=A_{k} x(n)+B_{k} u(n) \\
x(0)=\boldsymbol{X} \\
\Phi(u) \geq \alpha-\varepsilon \\
u^{-} \leq u(n) \leq u^{+}
\end{array}\right.
$$

then for $k$ large enough,

$$
J\left(u_{k}^{*}\right) \leq J\left(u^{*}\right)+\eta
$$

Remark 2: In fact, the solution of this problem is a sequence of $N_{k}$ control values $u(n) . u_{k}^{*}(t)$ is the piecewise constant function defined canonically from this sequence : $u_{k}^{*}\left(n T_{k}\right)=u(n)$.

Remark 3: This theorem claims that the solution of the discrete-time problem is an approximate solution of the continuous-time problem.

Proof: Let us denotes $u_{k}$ the discretized version of $u^{*}$, that is to say $u_{k}$ is a piecewise constant function $u_{k}(t)=$ $u\left(n T_{k}\right)$ if $n T_{k} \leq t<(n+1) T_{k}$.

Since $\mathbb{P}$ is supposed to be absolutely continuous with respect to Lebesgue measure, $\Phi$ is a continuous function of $u$. Therefore, for $k$ large enough, $\Phi\left(u_{k}\right) \geq \alpha-\varepsilon$. since $u_{k} \stackrel{k \rightarrow \infty}{\longrightarrow} u^{*}$ in $L^{2}([0, T])$ and $\Phi\left(u^{*}\right) \geq \alpha$. Therefore, $u_{k}$ satisfies constraints eq. (17d) and eq. (17e), and so $J\left(u_{k}^{*}\right) \leq$ $J\left(u_{k}\right)$. But $J\left(u_{k}\right) \stackrel{k \rightarrow \infty}{\longrightarrow} J\left(u^{*}\right)$ and therefore, for $k$ large enough, $J\left(u_{k}^{*}\right) \leq J\left(u^{*}\right)+\eta$.

\section{APPLICATION}

Let us consider for instance the following linear system in $\mathbb{R}^{2}$ :

$$
\dot{x}(t)=\left(\begin{array}{cc}
0 & 1 \\
0 & -1
\end{array}\right) x(t)+\left(\begin{array}{l}
0 \\
1
\end{array}\right) u(t)
$$

where $x(0)$ is a Gaussian variable $\mathcal{N}\left(0, \sigma^{2}\right)$.
Our objective is to maximize $E\left[x_{1}(T)\right]$ (equivalently $E\left[-x_{1}(T)\right] \longrightarrow \min _{u}$ ) where $T$ is a given finite time such that

$$
\mathbb{P}\left(-\varepsilon \leq x_{2}(T) \leq \varepsilon\right) \geq \alpha
$$

If $\sigma=0$, that is to say if the problem is a deterministic problem, one can solve the problem explicitly using the maximum principle of Pontryagin. It is easy to prove that (if $\varepsilon$ is not too large) there exists a time $0<\tau<T$ such that the optimal control is bang-bang and equal to one from $t=0$ to $\tau$ and is equal to minus one from $\tau$ to $T$. More precisely, one can calculate explicitly $\tau=\ln \left(\frac{1+(1+\varepsilon) \mathrm{e}^{T}}{2}\right)$ and the cost is equal to $x_{1}(T)=2 \tau-\varepsilon-T$. On Figure 1 , we plotted the solution of the problem with $T=3$ and $\varepsilon=\frac{1}{6}$ ( $x_{1}(t)$ in red, $x_{2}(t)$ in blue and $u(t)$ in black). In this case, the previous formula gives us $\tau \approx 2.5$ and $x_{2}(3) \approx 1.84$.

We solve the probabilistic case with $\sigma=10^{-4}$ and $\alpha=$ 0.9. We use the method described in Section IV. We calculate the discrete-time solution for $N_{1}=30$ and $N_{2}=300$.

The result for $N_{1}=30$ is plotted on Figure 2. One can verify that $x(t)$ have almost the same behavior as in the deterministic case because $\sigma$ is small and $\alpha$ is close to 1 . We have verified that statistically, when we simulate a large number of realization, the probability for $x_{2}(T)$ to be in the interval $[-\varepsilon,+\varepsilon]$ is equal to $\alpha=0.9$.

When $N_{2}=300, u_{300}$ becomes a good approximation of $u^{*}$. The result is shown on Figure 3 .

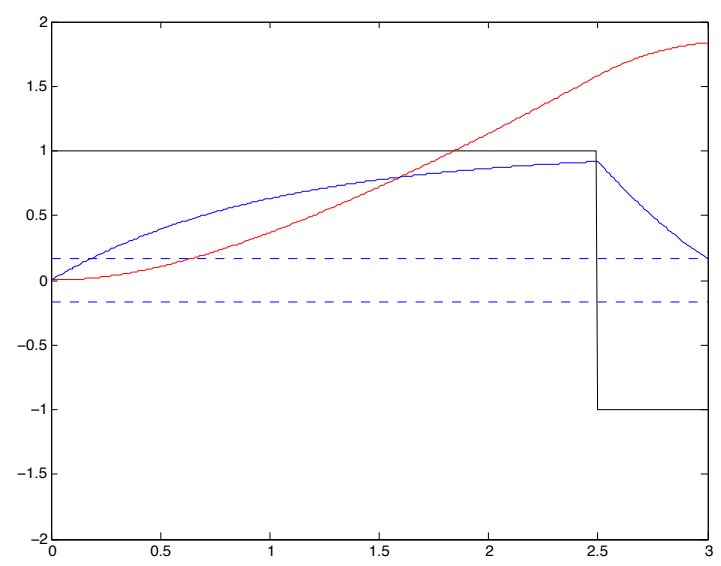

Fig. 1: Deterministic case, $T=3$

\section{CONCLUSION}

We presented a method to solve both discrete-time and continuous-time problems with chance constraints. We didn't suppose that Gaussian distribution is absolutely continuous w.r.t. Lebesgue measure. Therefore, we had to prove that, first of all, the problem remained convex and secondly, a numerical method could be applied to estimate the probability using a Monte Carlo method. We then used the discretetime problem to solve a continuous-time optimization problem with chance constraints. We proved that the discretetime solution converges to the continuous-time solution. We provided a very simple example to illustrate the method. 


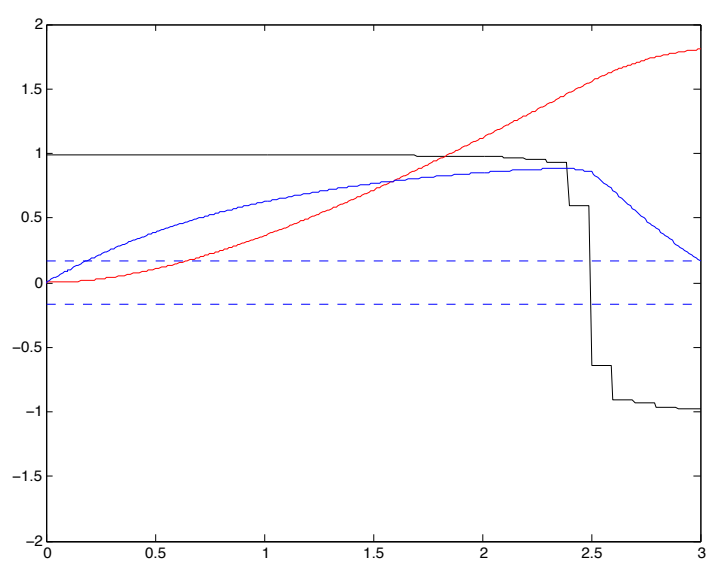

Fig. 2: Discrete problem, $N=30$

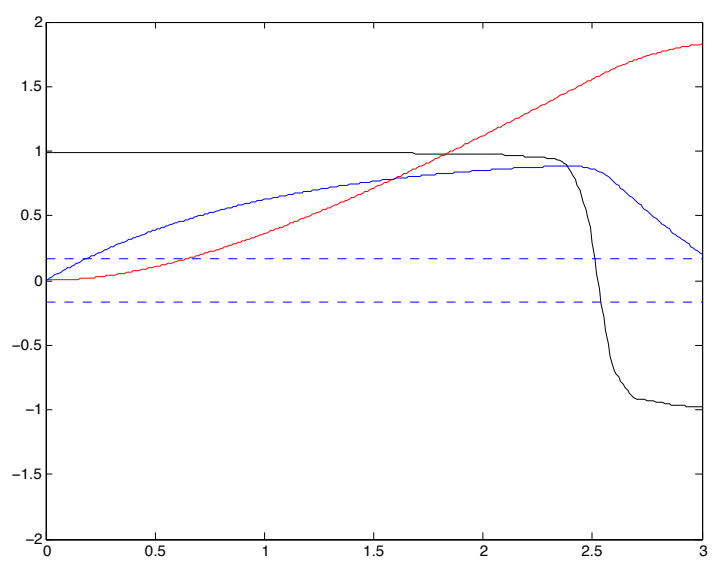

Fig. 3: Discrete problem, $N=300$

In future works (in preparation), we will propose a new method to solve directly the continuous-time problem and we will compare this new method to the method presented in this paper.

\section{REFERENCES}

[1] M. Farina, L. Giulioni, and R. Scattolini, "Stochastic linear model predictive control with chance constraints - a review," Journal of Process Control, vol. 44, pp. 53-67, aug 2016.

[2] F. Oldewurtel, C. N. Jones, A. Parisio, and M. Morari, "Stochastic model predictive control for building climate control," IEEE Transactions on Control Systems Technology, vol. 22, pp. 1198-1205, may 2014.

[3] F. Oldewurtel, C. N. Jones, A. Parisio, and M. Morari, "Stochastic model predictive control for building climate control," IEEE Transactions on Control Systems Technology, vol. 22, no. 3, pp. 1198-1205, 2014.

[4] J. Grosso, C. Ocampo-Martínez, V. Puig, and B. Joseph, "Chanceconstrained model predictive control for drinking water networks," Journal of Process Control, vol. 24, no. 5, pp. 504-516, 2014.

[5] A. Carvalho, Y. Gao, S. Lefevre, and F. Borrelli, "Stochastic predictive control of autonomous vehicles in uncertain environments," in 12th International Symposium on Advanced Vehicle Control, 2014.

[6] A. Prékopa, "Probabilistic programming," in Handbooks in Operations Research and Management Science, pp. 267-351, Elsevier BV, 2003.

[7] A. V. Fiacco and G. P. McCormick, Nonlinear programming: sequential unconstrained minimization techniques, vol. 4. New York: John Wiley and sons, inc., 1968.

[8] G. Schildbach, L. Fagiano, C. Frei, and M. Morari, "The scenario approach for stochastic model predictive control with bounds on closed- loop constraint violations," Automatica, vol. 50, no. 12, pp. 30093018, 2014.

[9] P. V. Mikheev, "Multidimensional gaussian probability density and its applications in the degenerate case," Radiophysics and Quantum Electronics, vol. 49, pp. 564-571, jul 2006.

[10] R. A. Horn and C. R. Johnson, Matrix analysis. Cambridge University Press (CUP), 1985. 